BMJ Open

Diabetes

Research

\& Care

\title{
Engaging South Asian women with type 2 diabetes in a culturally relevant exercise intervention: a randomized controlled trial
}

\author{
Alamelu Natesan, ${ }^{1}$ Vani C Nimbal, ${ }^{2}$ Susan L Ivey, ${ }^{1}$ Elsie J Wang, ${ }^{3}$ \\ Kristine A Madsen, ${ }^{1}$ Latha P Palaniappan ${ }^{3}$
}

To cite: Natesan $A$, Nimbal VC, Ivey SL, et al. Engaging South Asian women with type 2 diabetes in a culturally relevant exercise intervention: a randomized controlled trial. BMJ Open Diabetes Research and Care 2015;3:e000126. doi:10.1136/bmjdrc-2015000126

Received 22 June 2015 Revised 19 August 2015 Accepted 25 September 2015

\section{CrossMark}

\footnotetext{
${ }^{1}$ University of California Berkeley, School of Public Health, Berkeley, California, USA

${ }^{2}$ Palo Alto Medical Foundation Research Institute, Palo Alto, California, USA

${ }^{3}$ Stanford University School of Medicine, Palo Alto, California, USA
}

Correspondence to Dr Latha P Palaniappan; lathap@stanford.edu

\section{ABSTRACT}

Background: We examined the efficacy of a culturally relevant exercise program in improving glycated hemoglobin (HbA1c) among South Asian women with type 2 diabetes, compared with usual care.

Methods: This was a randomized controlled 8-week pilot study of Bollywood dance among South Asian women with type 2 diabetes. The intervention consisted of $1 \mathrm{~h}$ Bollywood dance classes offered twice per week. The primary outcome was change in $\mathrm{HbA1c}$. The effect of attendance on this outcome was also examined.

Results: The intervention group demonstrated a decrease in $\mathrm{HbA} 1 \mathrm{C}$ from baseline $(-0.18 \%(0.2 \%)$; $\mathrm{p}=0.018)$ compared with a non-significant increase in the usual care group $(+0.03 \%(0.2 \%))$; $p$ value for difference between groups was 0.032 . Participants attending at least 10 of 16 sessions had a statistically significant reduction in weight $(-0.69 \mathrm{~kg}(0.76 \mathrm{~kg}))$ compared with those attending fewer sessions (+0.86 kg (0.71 kg)).

Conclusions: These results support culturally relevant dance as a successful exercise intervention to promote $\mathrm{HbA1c}$ control, compared with usual care.

Trial registration number: NCT02061618.

\section{INTRODUCTION}

South Asians, including individuals originating from India, Pakistan, Sri Lanka, Nepal, and Bangladesh, are a rapidly growing ethnic group in the $\mathrm{USA}^{1}$ who are disproportionately affected by type 2 diabetes. ${ }^{2}$ While exercise is recommended in type 2 diabetes, South Asians report lower levels of physical activity than other racial/ethnic subgroups. ${ }^{3}$ South Asian immigrant women, in particular, are prone to low physical activity levels due to various perceived barriers, ${ }^{4} 5$ acculturative stressors, ${ }^{6}$ and cultural handicaps. These factors highlight the need for culturally tailored interventions in South Asian women with type 2 diabetes.

The limited number of studies investigating factors affecting South Asian immigrants'

\section{Key messages}

- Our randomized controlled trial examined the efficacy of a culturally relevant Bollywood dance exercise intervention in improving glycated hemoglobin $(\mathrm{HbA1c})$ among South Asian women with type 2 diabetes, compared with usual care (control).

- The intervention group demonstrated a statistically significant reduction in $\mathrm{HbA1c}$ from baseline compared with the control group.

- Participants attending at least $62 \%$ of sessions in the intervention had a statistically significant reduction in weight compared with those who attended fewer sessions.

- Participants who did not lose any weight in the intervention group still showed reductions in $\mathrm{HbA1c}$, while those experiencing no weight loss in the control group saw no reduction in $\mathrm{HbA} 1 \mathrm{c}$.

decisions on whether or not to engage in exercise, report that cultural dance is the only form of exercise chosen by this group to be both enjoyable and a motivator for activity. ${ }^{4} 78$ Cultural dance exercise programs can be conducted in community settings and provide a source of group cohesion and support as well as social interaction and enjoyment, all of which are motivating factors emphasized in qualitative studies among South Asian immigrants. ${ }^{4} 7$ Prior dance research interventions that have incorporated community partnerships, used an experienced female dance instructor from the same ethnic community to lead dance sessions, and included popular, traditional music selected by the participants themselves to help foster a sense of comfort and familiarity in highly sedentary immigrant and ethnic populations. ${ }^{9-12}$ This enabled participants to combat acculturative stressors and be motivated to engage in higher levels of physical activity.

South Asians have long enjoyed a rich culture involving a high-energy, popular 
dance style called Bollywood. ${ }^{13}$ Inspired by India's film industry, this art form is primarily modeled on traditional Indian dance forms (ie, classical and religious dance styles, including Kathak and Bhangra), and has grown to incorporate Western elements as well. ${ }^{13}$ Bollywood dance is also aerobically intense as it incorporates folk dances that utilize both upper and lower limb muscles, and is culturally familiar through the popular songs, moves, and shared symbolizations evoked. ${ }^{14}$ Both Bollywood dance and music are widely appreciated and watched in South Asian countries (not just India), as the native cinema industry is the primary source of entertainment and revenue ( 2.7 billion theater tickets sold in 2013) in this region of the world. ${ }^{13} 14$

This study proposes Bollywood dance as a physical activity intervention to help South Asian women manage their type 2 diabetes. We examined the efficacy of a Bollywood dance exercise program in improving diabetes outcomes as measured through glycated hemoglobin (HbA1c) in South Asian women, compared with usual care.

\section{METHODS}

The Culturally Relevant Exercise for Type 2 Diabetes (CURE-D) study was a randomized controlled 8-week pilot study of Bollywood dance in women with type 2 diabetes. The main outcome measure was change in HbA1c. This study was approved by the Palo Alto Medical Foundation Institutional Review Board; written informed consent was obtained from each participant.

\section{Participants}

Eligible South Asian women were identified from clinical data from the Palo Alto Medical Foundation electronic health records. Eligibility criteria included self-identified race/ethnicity as South Asian, age 18-85, and either type 2 diabetes as a physician-recorded diagnosis code (International Classification of Diseases (ICD) 9-CM code 250.X0 or 250.X2), HbAlc $>6.5 \%,{ }^{15}$ or use of oral antidiabetic medications.

Exclusion criteria included serious medical conditions that would prevent full participation or contraindicate physical activity, HbA1c $>11 \%$, insulin use, or limited English proficiency.

\section{Randomization}

Participants were randomly assigned to either intervention or wait-list control at the baseline visit via a blind drawing of sealed envelopes. Randomization envelopes were prepared by a researcher independent of the project using a random number generating algorithm. The wait-list control group received usual care, during which they carried on with their normal routines, until returning for an 8-week follow-up visit, after which they were offered the intervention.

\section{Measures}

All measures were collected at baseline and follow-up. Fingerstick serum HbAlc $(\% \mathrm{mmol} / \mathrm{mol})$ was measured using a validated Siemens DCA Vantage Analyzer. Anthropometric data (weight, height, blood pressure) were collected using standard methodology. Attendance was measured at each dance session. The intervention consisted of 16 dance sessions total.

\section{Intervention}

The 8-week intervention consisted of $1 \mathrm{~h}$ Bollywood dance classes offered twice per week at the India Community Center (ICC). ICC is a community center that serves the South Asian community in the San Francisco Bay Area. A partnership was created with the ICC to build a community-based participatory research partnership, and classes were taught at this site in order to provide a facility familiar to the community. Classes were taught by the lead Bollywood instructor at the community center. The exercise protocol consisted of $10 \mathrm{~min}$ of warm-up, $30 \mathrm{~min}$ of Bollywood exercise, $15 \mathrm{~min}$ of muscle resistance and weight training, and 5 min of cool-down stretches.

\section{Outcome}

The primary outcome measure was change in HbA1c from baseline to post-trial. Change in weight and body mass index (BMI) was also assessed. Furthermore, to evaluate dose effects of the physical activity intervention, we examined the effect of attendance on change in HbA1c.

\section{Statistical analysis}

Data were entered into a database (SAS V.9.3, Cary, North Carolina, USA) and screened for missing data and outliers. Non-parametric statistical comparisons of pre-trial and post-trial variables (ie, HbAlc, weight, BMI) were made using paired Wilcoxon signed-rank tests to examine within-group differences and Wilcoxon rank-sum tests to examine between-group differences. The number of dance sessions the intervention group attended was categorized into: $<10$ sessions and $\geq 10$ sessions. Change in weight from baseline to post-trial was categorized as: loss in weight versus no change in weight/gain in weight. Wilcoxon rank-sum tests were used to examine the association between attendance and weight change and change in HbAlc. All analyses were performed on participants who completed the study (per protocol analysis). An intention-to-treat (ITT) analysis was also conducted as a sensitivity test for the main outcomes (change in HbAlc, weight, and BMI) after imputing for missing post-trial values among dropouts using the last observation carried forward method (which were baseline values).

\section{RESULTS}

Twenty-eight women qualified, consented, and began the trial, of which 14 were randomized into the 
Table 1 Demographic and clinical characteristics at baseline

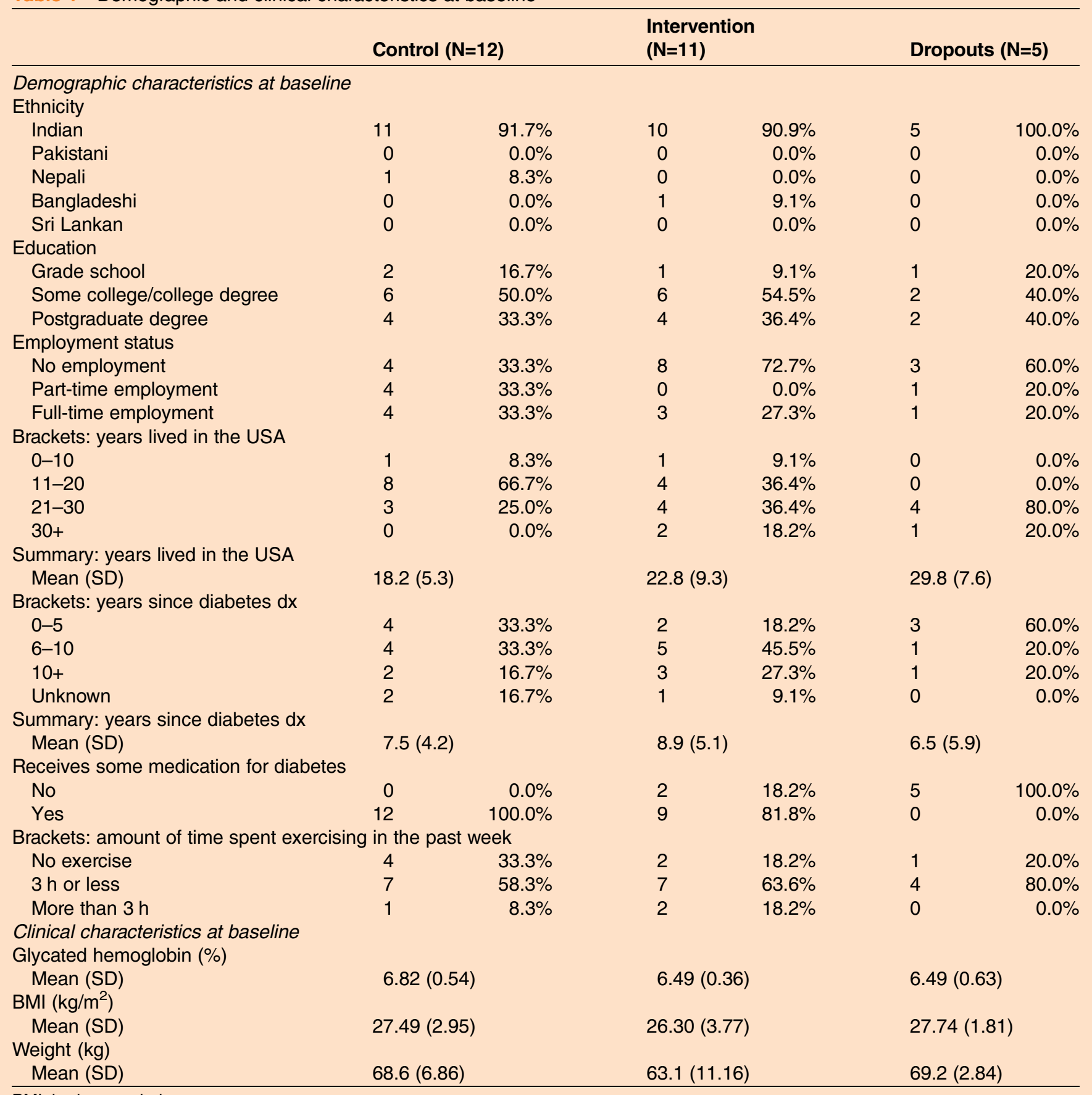

BMI, body mass index.

intervention group and 14 into the control group. Twenty-three participants $(82 \%)$ finished the 8-week trial (final sample $n=23$ ). There were similar rates of dropout between the two groups: 3 out of 14 (21.4\%) women dropped out of the intervention group, while 2 out of $14(14.3 \%)$ did so in the control group. Of the three women who dropped out of the intervention group, one dropped out before the first dance session due to health reasons, and the other two dropped out before the first session due to family emergencies requiring travel to India. Of the two women who dropped out of the control group, one dropped out before the final visit due to unspecified health reasons and the other before the final visit due to a change in her employment status.

Demographic and clinical characteristics were comparable between the intervention and control group at baseline (table 1). Mean HbAlc for the intervention and control group was $6.49 \%(\mathrm{SD} 0.4 \%$ ) and $6.82 \%$ (SD $0.5 \%$ ), respectively. The intervention group demonstrated a significant reduction in HbAlc $(-0.18 \%$ $(0.2 \%) ; \mathrm{p}=0.018)$ while no reduction was seen in the control group $(+0.03 \%(0.2 \%))$; $\mathrm{p}$ value for difference between groups was 0.032 . 
Table 2 Change in clinical characteristics (baseline to post-trial)

\begin{tabular}{|c|c|c|c|c|}
\hline \multirow{2}{*}{$\begin{array}{l}\text { Change in } \\
\text { clinical } \\
\text { characteristics }\end{array}$} & \multicolumn{2}{|c|}{ Control } & \multicolumn{2}{|c|}{ Intervention } \\
\hline & $\mathbf{N}$ & Mean (SD) & $\mathbf{N}$ & Mean (SD) \\
\hline $\begin{array}{l}\text { Change in } \\
\text { glycated } \\
\text { hemoglobin }(\%)^{*}\end{array}$ & 12 & $+0.03(0.24)$ & 11 & $-0.18(0.20) \dagger$ \\
\hline No weight loss & 7 & $+0.10(0.21)$ & 5 & $-0.14(0.27)$ \\
\hline $\begin{array}{l}\text { Some weight } \\
\text { loss }\end{array}$ & 5 & $-0.06(0.30)$ & 6 & $-0.22(0.13)$ \\
\hline Change in BMI & 12 & $-0.03(0.49)$ & 11 & $-0.11(0.41)$ \\
\hline $\begin{array}{l}\text { Change in weight } \\
\text { (in } \mathrm{kg} \text { ) }\end{array}$ & 12 & $-0.09(1.23)$ & 11 & $-0.26(1.02)$ \\
\hline
\end{tabular}

Compared with baseline, there were decreases in mean weight (intervention: $-0.26 \mathrm{~kg}(1.0 \mathrm{~kg})$, control: $-0.09 \mathrm{~kg}(1.2 \mathrm{~kg}))$ and BMI (intervention: $-0.11 \mathrm{~kg} / \mathrm{m}^{2}$ $\left(0.4 \mathrm{~kg} / \mathrm{m}^{2}\right)$, control: $\left.-0.03 \mathrm{~kg} / \mathrm{m}^{2}\left(0.5 \mathrm{~kg} / \mathrm{m}^{2}\right)\right)$ in both groups post-trial, but this decrease was more pronounced in the intervention group. However, results were not significant in either group. Among the six participants who lost weight in the intervention group, mean HbA1c decreased by $-0.22 \%$ (SD 0.1\%), while the five participants who lost weight in the control group had a mean HbA1c decrease of $-0.06 \%$ (SD $0.3 \%)$, although this difference was not statistically significant (table 2). Participants who did not lose weight in the intervention group still experienced a reduction in mean HbAlc $(-0.14 \%(0.3 \%))$, while those in the control group did not $(+0.1 \%(0.2 \%))$. Furthermore, participants attending at least 10 sessions (8/11 participants) had a statistically significant $(\mathrm{p}=0.032)$ reduction in weight $(-0.69 \mathrm{~kg}(0.8 \mathrm{~kg}))$ compared with those attending fewer than 10 sessions (weight: $+0.86 \mathrm{~kg}$ $(0.7 \mathrm{~kg}))$.
In this randomized controlled trial, there were no statistically significant baseline differences in $\mathrm{HbAlc}$, weight, or BMI between the intervention and control groups ( $\mathrm{p}$ values were $0.094,0.23$, and 0.31 , respectively). However, in order to address potential variability in baseline characteristics between the control and intervention groups, we conducted a sensitivity analysis on change in HbA1c, stratified by baseline HbA1c levels as well as baseline BMI and weight (table 3). The descriptive data consistently showed that the intervention group had reductions in HbAlc levels from baseline to posttrial across different levels of baseline characteristics, while the control group showed no reductions in HbA1c. However, given the very small sizes within the stratifications, many of these post hoc substratified results did not reach statistical significance.

The ITT analysis, conducted as a sensitivity test, showed similar results to that of the per protocol analysis. The intervention group demonstrated a significant reduction in $\mathrm{HbAlc}(-0.14 \%(0.2 \%))$ while no reduction was seen in the control group $(+0.03 \%(0.2 \%))$; $\mathrm{p}$ value for difference between groups was 0.029 . In addition, the ITT analysis also showed that both groups had non-significant reductions in mean weight (intervention: $-0.21 \mathrm{~kg}(0.9 \mathrm{~kg})$, control: $-0.08 \mathrm{~kg}(1.1 \mathrm{~kg}))$ and BMI (intervention: $\quad-0.09 \mathrm{~kg} / \mathrm{m}^{2} \quad\left(0.4 \mathrm{~kg} / \mathrm{m}^{2}\right)$, control: $\left.-0.02 \mathrm{~kg} / \mathrm{m}^{2}\left(0.5 \mathrm{~kg} / \mathrm{m}^{2}\right)\right)$ from baseline to post-trial, with a greater decrease among the intervention group.

\section{CONCLUSIONS}

This was the first randomized controlled trial evaluating Bollywood dance for South Asian women with type 2 diabetes. Bollywood dance exercise classes two times per week for 8 weeks showed significant reduction in HbA1c for the intervention group. The intervention group demonstrated a statistically significant decrease in HbA1c from baseline compared with the control group. These results further strengthen the notion that culturally sensitive dance is a successful exercise intervention to promote HbAlc control compared with usual care.

Table 3 Change in $\mathrm{HbA1c}$ (baseline to post-trial) accounting for baseline characteristics

\begin{tabular}{|c|c|c|c|c|}
\hline \multirow[b]{2}{*}{ Change in clinical characteristics } & \multicolumn{2}{|c|}{ Control } & \multicolumn{2}{|c|}{ Intervention } \\
\hline & $\overline{\mathbf{N}}$ & Mean (SD) & $\overline{\mathbf{N}}$ & Mean (SD) \\
\hline \multicolumn{5}{|l|}{ Change in $\mathrm{HbA} 1 \mathrm{c}(\%)$} \\
\hline Baseline $\mathrm{HbA} 1 \mathrm{c} \leq 6.6 \%$ & 4 & $+0.25(0.30)$ & 8 & $-0.14(0.19)$ \\
\hline Baseline $\mathrm{HbA} 1 \mathrm{c}>6.6 \%$ & 8 & $+0.04(0.23)$ & 3 & $-0.30(0.20)$ \\
\hline \multicolumn{5}{|l|}{ Change in $\mathrm{HbA} 1 \mathrm{c}(\%)$} \\
\hline Baseline $\mathrm{BMI} \leq 27 \mathrm{~kg} / \mathrm{m}^{2}$ & 4 & $+0.25(0.38)$ & 7 & $-0.14(0.21)$ \\
\hline Baseline $\mathrm{BMI}>27 \mathrm{~kg} / \mathrm{m}^{2 \star}$ & 8 & $+0.04(0.17)$ & 4 & $-0.25(0.19)$ \\
\hline \multicolumn{5}{|l|}{ Change in $\mathrm{HbA} 1 \mathrm{c}(\%)$} \\
\hline Baseline weight $<68 \mathrm{~kg}$ & 6 & $+0.03(0.29)$ & 7 & $-0.14(0.21)$ \\
\hline Baseline weight $\geq 68 \mathrm{~kg}$ & 6 & $+0.03(0.20)$ & 4 & $-0.25(0.19)$ \\
\hline
\end{tabular}

${ }^{*} p<0.05$ for change in $\mathrm{HbA} 1 \mathrm{c}$ between intervention and control group.

$\mathrm{BMI}$, body mass index; HbA1c, glycated hemoglobin. 
A fairly high proportion of participants, who were enrolled in the trial, completed the trial (retention rate: $82 \%)$. Furthermore, the average rate of classes attended by the intervention group was also fairly high (attendance rate: $69 \%$ ); both rates were comparable to those of other culturally sensitive dance intervention studies conducted in Latino and African-American populations. ${ }^{11} 16$ High participation rates in dance classes are an important predictor of improvement in anthropometric outcomes. ${ }^{11}$ Our study supported this finding, showing that participants attending at least $62 \%$ of sessions had statistically significant reductions in weight compared with those who attended fewer.

Weight reduction is known to improve glycemic control and insulin resistance. Therefore, weight loss might mediate the effect of physical activity on glycemic control, as measured by reduction in HbAlc. While we did find that the intervention group had larger decreases in weight, those who did not lose any weight in the intervention group still had a reduction in HbA1c, while those who did not lose weight in the control group saw no reduction. Prior research has shown that resistance training, by increasing muscle mass, can contribute to greater blood glucose uptake. ${ }^{17}$ Since our exercise regimen included resistance training, women who experienced no weight loss may have improved muscle mass and thus glycemic control.

While this study serves as proof-of-concept for culturally tailored dance for South Asian women, there are limitations that should be taken into account when interpreting results. The dance classes were all held in the daytime on a weekday, therefore many women working full time were unable to enroll in the program. Furthermore, although this was a randomized trial, we found some variability in baseline characteristics in each of the groups due to the small sample size. An exploratory analysis showed that the intervention group consistently had reductions in HbA1c levels across different levels of baseline characteristics, while the control group showed no reductions. These results are mostly descriptive because we lacked power in this pilot study to control for baseline characteristics.

Additionally, we had two dropouts in the control group and three in the intervention group. The reasons for the dropouts indicate that they were not due to the intervention itself, as does the observation that all three dropouts in the intervention group occurred before the actual dance intervention began. We also conducted an ITT sensitivity analysis to ensure that dropouts did not considerably affect the random treatment allocation. While our options for imputing missing post-trial values for the dropouts were limited and imperfect, the results of the ITT analysis remained robust and comparable to that of the per protocol analysis.

Another limitation of our study was that we were unable to fully account for the type, dosing, and timing of diabetic medications. However, since we excluded insulin users from our trial, our participants may be earlier in their disease progression when lifestyle change may have a more potent impact on preventing consequences of diabetes. We found that $75 \%(9 / 12)$ of the control group and $91 \%(10 / 11)$ of the intervention group had a HbA1c value of $7 \%$ or less at baseline further suggesting that a large proportion of our participants in both groups were already within glycemic targets recommended by the American Diabetes Association at baseline. Other studies have demonstrated that education, lifestyle changes, and health coaching can have impacts on HbAlc in the similar range of medication therapy $\left(0.5-1.5 \%\right.$ decreases in HbAlc) ${ }^{18-20}$ We do not have data on the type and dosage of medications and were unable to compare these characteristics between the control and intervention group, although this would be interesting to examine in a larger study. However, we do know that $82 \%(9 / 11)$ of the intervention group and $100 \%$ of the control group were receiving some type of oral medication prior to the study. Given that this intervention was only 8 weeks long, we do not expect that many participants had their medication dosages titrated during the short course of the intervention and therefore feel confounding effects of this on the outcome are less likely. Finally, the sample size in each of the groups was small, thus limiting our power to detect differences between groups. However, this was a pilot proof-of-concept study whose implications must be further examined in a larger randomized control trial.

Implications from this study will be of use in examining the sustainability of a Bollywood dance exercise intervention in a more rigorous randomized controlled trial with a larger sample size, and with additional control groups that are engaged in other types of physical activity. The findings should also be used in the real world to design and implement dance programs that improve health outcomes in the South Asian community.

Acknowledgements The authors would like to thank the India Community Center (ICC) for supporting this study and providing their facilities for the dance classes. Namely, they would like to thank Tanuja Bahal, Pragati Grover, and the entire executive board of the ICC for their encouragement and assistance. They would also like to thank the fitness director at the ICC, Shelly Rojas, for teaching all of the dance sessions. They would also like to thank all of the researchers of the DISCOVeR team at the Palo Alto Medical Foundation Research Institute who volunteered at their baseline and final visits: Kristen Azar, Marina Dolginsky, Edith Gamboa, Katie Hastings, Martina Li, Laura Nolting, Cecelia Peterson, Jia Pu, and Chrystal Rabino. In closing, they would like to thank the UC Berkeley-UCSF Joint Medical Program for providing continued support throughout each phase of the study.

Contributors AN wrote the manuscript, collected, and analyzed data. VCN wrote the manuscript and conducted statistical analyses. SLI, KAM and LPP contributed to the discussion and reviewed/edited the manuscript. EJW reviewed/edited the manuscript and researched data.

Funding UC Berkeley-UCSF Joint Medical Program Schoeneman Summer Stipend.

Competing interests None declared.

Patient consent Obtained. 
Ethics approval Palo Alto Medical Foundation Institutional Review Board.

Provenance and peer review Not commissioned; externally peer reviewed.

Data sharing statement In addition to participant demographic, anthropometric, and diabetes outcomes data, information on participant quality of life, exercise enjoyment, and satisfaction were also collected. All de-identified data can be provided by researchers.

Open Access This is an Open Access article distributed in accordance with the Creative Commons Attribution Non Commercial (CC BY-NC 4.0) license, which permits others to distribute, remix, adapt, build upon this work noncommercially, and license their derivative works on different terms, provided the original work is properly cited and the use is non-commercial. See: http:// creativecommons.org/licenses/by-nc/4.0/

\section{REFERENCES}

1. Hoeffel EM, Rastogi S, Kim MO, et al. The Asian population: 2010. US Department of Commerce, Economics and Statistics Administration, US Census Bureau, 2012.

2. Center for Disease Control and Prevention. National diabetes statistics report: estimates of diabetes and its burden in the United States, 2014. Atlanta, GA: US Department of Health and Human Services, 2014

3. Ye J, Rust G, Baltrus $\mathrm{P}$, et al. Cardiovascular risk factors among Asian Americans: results from a National Health Survey. Ann Epidemiol 2009;19:718-23.

4. Jepson R, Harris FM, Bowes A, et al. Physical activity in South Asians: an in-depth qualitative study to explore motivations and facilitators. PLOS ONE 2012;7:e45333.

5. Bajaj S, Jawad F, Islam N, et al. South Asian women with diabetes: psychosocial challenges and management: consensus statement. Indian J Endocrinol Metab 2013;17:548.

6. Hine $\mathrm{C}$, Fenton $\mathrm{S}$, Hughes $\mathrm{AO}$, et al. Coronary heart disease and physical activity in South Asian women: local context and challenges. Health Educ J 1995;54:431-43.

7. Kandula NR, Patel Y, Dave S, et al. The South Asian Heart Lifestyle Intervention (SAHELI) study to improve cardiovascular risk factors in a community setting: design and methods. Contemp Clin Trials 2013;36:479-87.
8. Vahabi M, Damba C. A feasibility study of a culturally and gender-specific dance to promote physical activity for South Asian immigrant women in the greater Toronto area. Womens Health Issues 2015;25:79-87.

9. Flores R. Dance for health: improving fitness in African American and Hispanic adolescents. Public Health Rep 1995;110:189.

10. Jain S, Brown DR. Cultural dance: an opportunity to encourage physical activity and health in communities. Am J Health Educ 2001;32:216-22.

11. Murrock CJ, Gary FA. Culturally specific dance to reduce obesity in African American women. Health Promot Pract 2010;11:465-73.

12. Ramachandran A, Snehalatha C, Mary S, et al. The Indian Diabetes Prevention Programme shows that lifestyle modification and metformin prevent type 2 diabetes in Asian Indian subjects with impaired glucose tolerance (IDPP-1). Diabetologia 2006;49:289-97.

13. Dudrah RK. Bollywood: sociology goes to the movies. Sage, 2006.

14. Mundra V. Obesity management: dancing to the Bhangra beat. Indian J Endocrinol Metab 2012;16:868-9.

15. American Diabetes Association. Diagnosis and classification of diabetes mellitus. Diabetes Care 2013;36(Suppl 1):S67-74.

16. Martyn-Nemeth PA, Vitale GA, Cowger DR. A culturally focused exercise program in hispanic adults with type 2 diabetes: a pilot study. Diabetes Educ 2010;36:258-67.

17. Colberg SR, Sigal RJ, Fernhall B, et al. Exercise and type 2 diabetes: the American College of Sports Medicine and the American Diabetes Association: joint position statement. Diabetes Care 2010;33:e147-67.

18. Franz MJ, Boucher JL, Rutten-Ramos S, et al. Lifestyle weight-loss intervention outcomes in overweight and obese adults with type 2 diabetes: a systematic review and meta-analysis of randomized clinical trials. J Acad Nutr Diet 2015;115:1447-63.

19. Attridge M, Creamer J, Ramsden M, et al. Culturally appropriate health education for people in ethnic minority groups with type 2 diabetes mellitus. Cochrane Database Syst Rev 2014;9: CD006424.

20. Philis-Tsimikas A, Fortmann A, Lleva-Ocana L, et al. Peer-led diabetes education programs in high-risk Mexican Americans improve glycemic control compared with standard approaches: a Project Dulce promotora randomized trial. Diabetes Care 2011;34:1926-31. 